\title{
GENERALIZED STIELTJES FUNCTIONS AND THEIR EXACT ORDER
}

\author{
D. KARP AND E. PRILEPKINA
}

Abstract. The paper surveys the basic properties of generalized Stieltjes functions including some new ones. We introduce the notion of the exact Stieltjes order and give a criterion of exactness along with simple sufficient conditions and some prototypical examples. The paper includes an appendix, where we define the left sided Riemann-Liouville and the right sided Kober-Erdélyi fractional integrals of measures supported on half axis and give inversion formulas for them.

Mathematics subject classification (2010): 26A48, 26A33, 44A15.

Keywords and phrases: Generalized Stieltjes transform, generalized Stieltjes function, fractional integral, fractional derivative, exact Stieltjes order.

\section{REFERENCES}

[1] G.E. Andrews, R. Askey and R. Roy, Special functions, Cambridge University Press, 1999.

[2] G.A. BAKer JR. And P. Graves-Morris, Padé Aproximants. Encyclopedia of Mathematics and its Applications, vol. 59. Cambridge University Press, Cambridge, 1996.

[3] C. Berg, Quelques remarques sur le cône de Stieltjes, in Séminaire de Théorie du Potentiel, Paris, No.5, in: F. Hirsch, G. Mokobodzki (Eds.), Lecture Notes in Mathematics, vol.814, Springer, Berlin, Heidelberg, New York, 1980, 70-79.

[4] V.I. BogacheV, Measure Theory, Volume I, Springer, 2007.

[5] R.B. BurCKel, An introduction to clasical complex analysis, vol.1, Birkhaüser Verlag Basel, 1979.

[6] A. ByRne AND E.R. Love, Complex inversion theorems for generalized Stieltjes transforms, J. Austral. Math. Soc. 18 (1974), 328-358.

[7] M. CARTER B. VAn BRUnt, The Lebesgue-Stieltjes integral. A practical Introduction, Springer, 2000.

[8] A. Cuyt, V.B. Petersen, B. Verdonk, H. Waadeland, W.B. Jones, Handbook of Continued Fractions for Special Functions, Springer, 2008.

[9] K. Diethelm, The Analysis of Fractional Differential Equations, Lecture Notes in Mathematics 2004, Springer, 2010.

[10] J. GILEWICZ, 100 years of improvements of bounding properties of Padé approximants to the Stieltjes functions: One-point, two-point and N-point Padé approximants, Applied Numerical Mathematics 60 (2010), 1320-1331.

[11] F. HiRSCH, Intégrales de résolvantes et calcul symbolique, Annales de l'insitut Fourier 22, 4 (1972), 239-264.

[12] I.I. HiRschman And D.V. Widder, The convolution transform, Princeton Mathematical Series, no.20, M. Morse and A.W. Tucker (eds.), Princeton University Press, 1955.

[13] A.M. Jerbashian, Functions of $\alpha$-bounded type in the half-plane, Springer, 2005.

[14] D. Karp AND E. PRILEPKINA, Generalized Stieltjes transforms: basic aspects, 2011, preprint arXiv:1111.4271v1.

[15] D. KARP AND E. PRILEPKINA, Hypergeometric functions as generalized Stieltjes transforms, Journal of Mathematical Analysis and Applications, 393, 2 (2012), 348-359.

[16] A.A. Kilbas, H.M. SRivastava And J.J. Trujillo, Theory and application of fractional differential equations, North-Holland Mathematical Studies, 204, 2006.

[17] V.S. Kiryakova, Generalized Fractional Calculus and Applications, Pitman Research Notes in. Math. Series No. 301, Longman Group UK Ltd., 1994. 
[18] S. Koumandos, H. L. Pedersen, Completely monotonic functions of positive order and asymptotic expansions of the logarithm of Barnes double gamma function and Euler's gamma function, J. Math. Anal. Appl. 355 (2009), 33-40.

[19] M.G. KREIN AND A.A. Nudelman, The Markov moment problem and extremal problems. Translations of mathematical monographs, v. 50, AMS, Providence, R.I., 1977.

[20] J.L. LóPeZ AND C. Ferreira, Asymptotic Expansions of Generalized Stieltjes Transforms of Algebraically Decaying Functions, Studies in Applied Math. 108 (2002), 187-215.

[21] E.R. Love AND A. B YRne, Real Inversion theorems for generalized Stieltjes transform, J. London Math. Soc. (2) 22 (1980), 285-306.

[22] E.R. Love AND A. BYRnE, Real Inversion theorems for generalized Stieltjes transform II, Math. Proc. Camb. Phil. Soc. 92 (1982), 275-291.

[23] K.S. Miller, B. Ross, Introduction to fractional calculus, John Wiley and Sons, 1993.

[24] H. Pedersen, Completely monotonic functions related to logarithmic derivatives of entire functions, Analysis and Applications 9, 4 (2011), 409-419.

[25] I. Podlubny, Fractional differential equations, Mathematics in Science and Engineering, vol.198, Academic Pres, 1999.

[26] H.L. Royden, F.M. FitzPatrick, Real Analysis, Fourth Edition, Pearson Education, 2010.

[27] S.G. Samko, A.A. Kilbas, O.I. Marichev, Fractional Integrals and Derivatives: Theory and Applicatons, Gordon and Breach Science Publishers, 1993.

[28] R.L. Schilling, Measures, integrals and martingales, Cambridge University Press, 2005.

[29] R.L. SChilling, R. SONG Z. VondračEK, Bernstein Functions. Theory and Applications, Walter de Gruyter, Studies in Mathematics, 37, 2010.

[30] J.H. SChWARZ, The generalized Stieltjes transform and its inverse, J. of Math. Phys. 46 (2005), 013501.

[31] A.D. Sokal, Real-variables characterization of generalized Stieltjes functions, Expo. Math. 28 (2010), 179-185.

[32] T.J. Stieltjes, Recherches sur les fractions continues, Ann. Fac. Sci. Toulouse 8 (1894), 1122; 9 (1895), 5-47. (Reprinted, together with an English translation, in: T.J. Stieltjes, Oeuvres Complètes/Collected Papers, vol.II, 1993, Springer, Berlin, 401-566, 609-745).

[33] D.B. Sumner, An inversion formula for the generalized Stieltjes transform, Bull. Amer. Math. Soc. 55 (1949), 174-183.

[34] The On-Line Encyclopedia of Integer Sequences, http://oeis.org/.

[35] D.V. WIDDER, The Laplace transform, Princeton University Press, 1946.

[36] D.V. WIDDER, The Stieltjes transform, Trans. of Amer.Math.Soc. 43 (1938), 7-60.

[37] O. YÜREKLI, A theorem on the generalized Stieltjes transfrom and its applications, Journal of Mathematical Analysis and Its Applications 168 (1992), 63-71. 